Supporting Information

\title{
Ultrafast Detection of Exosomal RNAs via Cationic Lipoplex Nanoparticles in a Micro-Mixer Biochip for Cancer Diagnosis
}

Yunchen Yang ${ }^{l}$, Eric Kannisto ${ }^{2}$, Santosh K. Patnaik ${ }^{2}$, Mary E. Reid ${ }^{3}$, Lei Li ${ }^{4}$, Yun Wu $^{l, *}$

${ }^{1}$ Department of Biomedical Engineering, University at Buffalo, The State University of New York, 332 Bonner Hall, Buffalo, NY 14260, United States

${ }^{2}$ Department of Thoracic Surgery, Roswell Park Comprehensive Cancer Center, Elm and Carlton Street, Buffalo, NY 14263, United States

${ }^{3}$ Department of Medicine, Roswell Park Comprehensive Cancer Center, Elm and Carlton Street, Buffalo, NY 14263, United States

${ }^{4}$ School of Mechanical and Materials Engineering, Washington State University, Pullman, WA 99164, United States

* Corresponding Author

Email: ywu32@buffalo.edu

Phone: (716) 645-8498

Fax: (716) 645-2207 


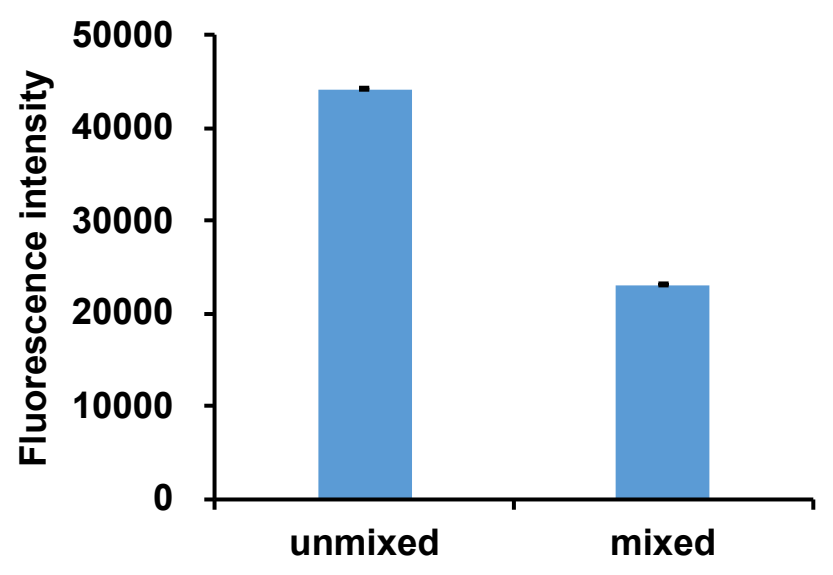

Figure S1. Evaluation of the mixing performance of the micro-mixer biochip. The fluorescence intensity of Rhodamine B in the solution collected at the outlet of the micro-mixer biochip was $\sim 50 \%$ of that of the original, unmixed solution, indicating that an effective mixing was achieved by the micro-mixer biochip. 
a)

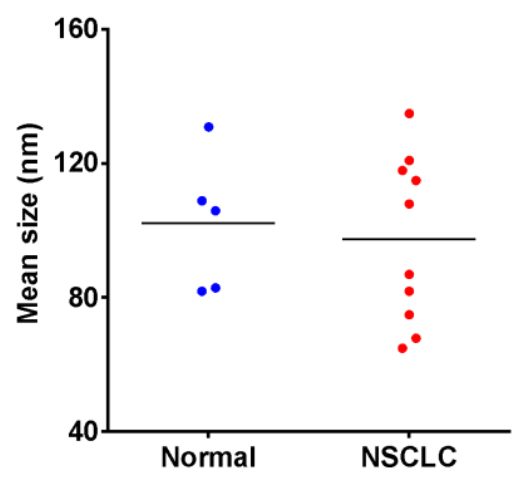

c)

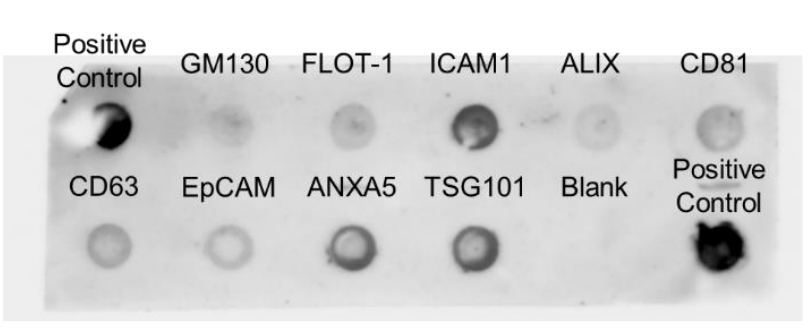

b)

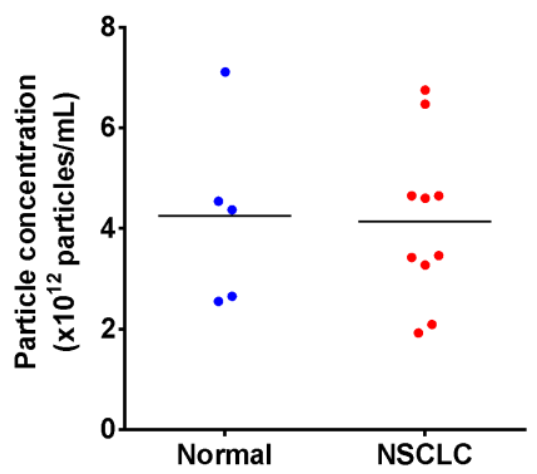

d)

Late stage NSCLC patient

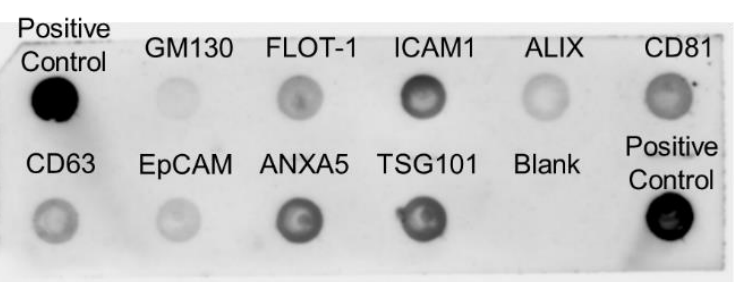

Figure S2. Characterization of human serum derived exosomes. The sizes (a) and number concentrations (b) of exosomes. No significant difference was observed between normal controls and NSCLC patients. Exosomes isolated from the serum samples of a normal control (c) and a late stage lung cancer patient (d) showed strong expression of exosome markers (such as CD81, CD63, ICAM1, ANXA5 and TSG101) and weak expression of GM130 (a cis-Golgi marker as the negative control), demonstrating exosomes used in this study had good purity. 


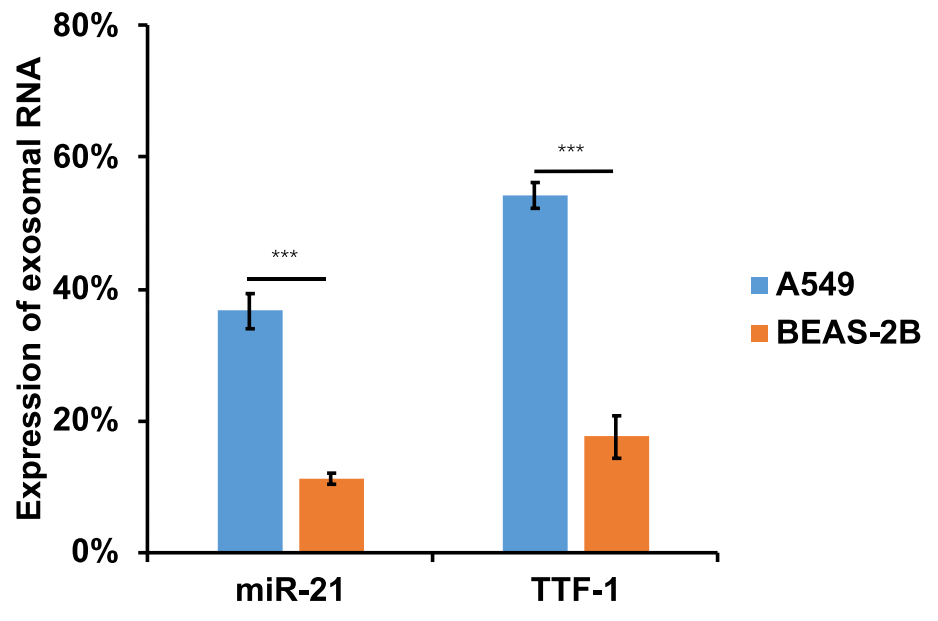

Figure S3. Detection of exosomal miR-21 and TTF-1 mRNA at CLP-MBs: exosomes number ratio of 2:1. The exosome concentration was $8 \times 10^{10}$ exosomes $/ \mathrm{mL}$ and the CLP-MBs concentration was $1.6 \times 10^{11}$ particles $/ \mathrm{mL}$ to achieve CLP-MBs: exosomes number ratio of 2:1. The biochips with 10 micro-mixer units were used. Significantly higher levels of miR-21 and TTF-1 mRNA were detected in A549 cell-derived exosomes than BEAS-2B cell-derived exosomes. However, compared with results obtained at CLP-MBs: exosomes number ratio of $1: 5$, the differences were smaller, indicating a lower sensing sensitivity. 


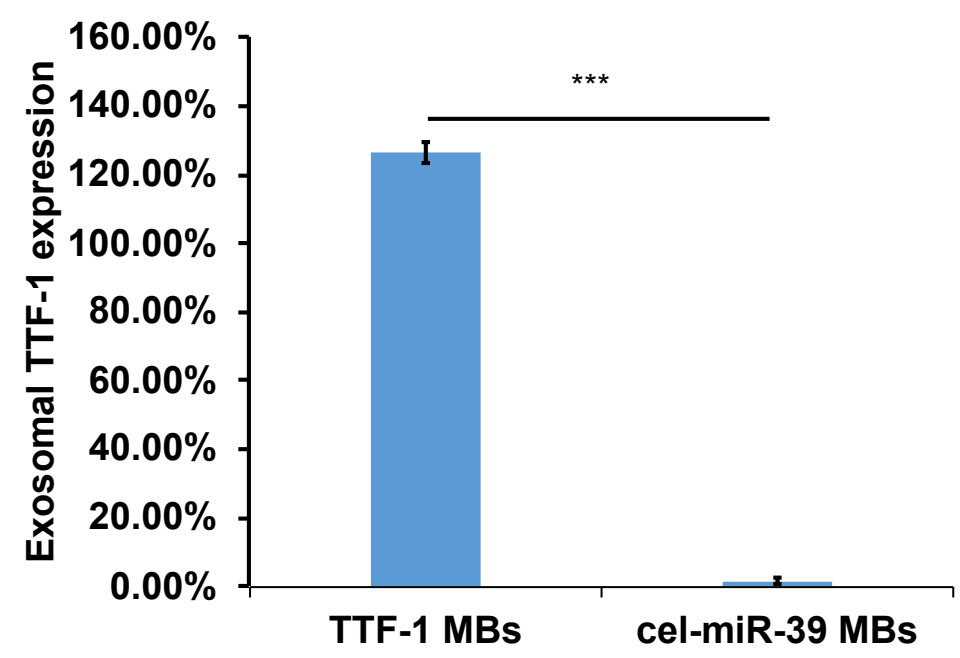

Figure S4. The mCLN assay showed excellent sensing specificity. Cy5-TTF-1 MBs or Cy5-celmiR-39 MBs (5'-[Cyanine5]-CGCGATCCAAGCTGATTTACACCCGGTGAG ATCGCG[BHQ3]-3') were encapsulated in CLP to detect TTF-1 mRNA levels in A549 cell-derived exosomes. The concentration of exosomes was $8 \times 10^{10}$ exosomes $/ \mathrm{mL}$. The concentration of CLP containing Cy5-cel-miR-39 MBs or Cy5-TTF-1 MBs was $1.6 \times 10^{10}$ particles $/ \mathrm{mL}$ to make CLPMBs: exosomes number ratio of 1:5. The biochips with 10 micromixer units were used. Compared with Cy5-TTF-1 MBs, little fluorescence was observed with Cy5-cel-miR-39 MBs, indicating great sensing specificity of the mCLN assay. 
Table S1 Characteristics of human subjects

\begin{tabular}{|c|c|c|c|c|c|c|}
\hline $\begin{array}{c}\text { Sample } \\
\text { ID }\end{array}$ & Disease & Stage & Gender & Age & $\begin{array}{c}\text { Exosome } \\
\text { Exosome } \\
\text { size (nm) }\end{array}$ & $\begin{array}{c}\text { number } \\
\text { concentration } \\
\mathbf{( 1 0}^{\mathbf{1 2}} \\
\text { exosomes/mL) }\end{array}$ \\
\hline 1 & Normal & NA & F & $65-69$ & 83 & 2.66 \\
\hline 2 & Normal & NA & F & $65-69$ & 82 & 4.55 \\
\hline 3 & Normal & NA & M & $65-69$ & 106 & 7.12 \\
\hline 4 & Normal & NA & M & $75-79$ & 109 & 2.56 \\
\hline 5 & Normal & NA & F & $65-69$ & 131 & 4.38 \\
\hline 6 & Adenocarcinoma & 1A & F & $60-64$ & 82 & 3.28 \\
\hline 7 & Adenocarcinoma & 1A & M & $65-69$ & 65 & 6.48 \\
\hline 8 & Adenocarcinoma & 2B & F & $70-74$ & 115 & 3.47 \\
\hline 9 & Adenocarcinoma & 1A & F & $70-74$ & 68 & 6.76 \\
\hline 10 & Adenocarcinoma & 1B & F & $60-64$ & 121 & 4.61 \\
\hline 11 & Squamous cell carcinoma & 3A & M & $60-64$ & 135 & 1.93 \\
\hline 12 & Adenocarcinoma & 4 & F & $75-80$ & 75 & 4.66 \\
\hline 13 & Adenocarcinoma & 3A & F & $65-69$ & 108 & 3.43 \\
\hline 14 & Squamous cell carcinoma & 4 & M & $55-59$ & 87 & 2.10 \\
\hline 15 & Adenocarcinoma & 3A & M & $70-74$ & 118 & 4.66 \\
\hline
\end{tabular}

